\title{
New results with Swift on Supergiant Fast X-ray Transients
}

\author{
L. Sidoli ${ }^{*},{ }^{a}$ P. Romano, ${ }^{b}$ L. Ducci, ${ }^{a c}$ A. Paizis, ${ }^{a}$ S. Vercellone, ${ }^{b}$ G. Cusumano,,${ }^{b}$ V. La \\ Parola, ${ }^{b}$ V. Mangano, ${ }^{b}$ J.A. Kennea, ${ }^{d}$ D.N. Burrows, ${ }^{d}$ H.A. Krimm, ${ }^{e f g}$ N. Gehrels, ${ }^{g}$ \\ V. Sguera, ${ }^{h i}$ A. Bazzano ${ }^{i}$ \\ ${ }^{a}$ INAF, Istituto di Astrofisica Spaziale e Fisica Cosmica, \\ Via E. Bassini 15, I-20133 Milano, Italy \\ ${ }^{b}$ INAF, Istituto di Astrofisica Spaziale e Fisica Cosmica, \\ Via U. La Malfa 153, I-90146 Palermo, Italy \\ ${ }^{c}$ Dipartimento di Fisica e Matematica, Università degli Studi dell'Insubria, \\ Via Valleggio 11, I-22100 Como, Italy \\ ${ }^{d}$ Department of Astronomy and Astrophysics, Pennsylvania State University, \\ University Park, PA 16802, USA \\ ${ }^{e}$ CRESST/Goddard Space Flight Center, Greenbelt, MD, USA \\ ${ }^{f}$ Universities Space Research Association, Columbia, MD, USA \\ ${ }^{g}$ NASA/Goddard Space Flight Center, Greenbelt, MD 20771, USA \\ ${ }^{h}$ INAF, Istituto di Astrofisica Spaziale e Fisica Cosmica, \\ Via Gobetti 101, I-40129 Bologna, Italy \\ iINAF, Istituto di Astrofisica Spaziale e Fisica Cosmica, \\ Via Fosso del Cavaliere 100, I-00133, Roma, Italy \\ E-mail: sidoli@iasf-milano.inaf.it
}

\begin{abstract}
We report here on the most recent results obtained on a new class of High Mass X-ray Binaries, the Supergiant Fast X-ray Transients. Since October 2007, we have been performing a monitoring campaign with Swift of four SFXTs (IGR J17544-2916, XTE J1739-302, IGR J16479-4514 and the X-ray pulsar AX J1841.0-0536) for about 1-2 ks, 2-3 times per week, allowing us to derive the previously unknown long term properties of this new class of sources (their duty cycles, spectral properties in outbursts and out-of-outbursts, temporal behaviour). We also report here on additional Swift observations of two SFXTs which are not part of the monitoring: IGR J184830311 (observed with Swift/XRT during a whole orbital cycle) and SAX J1818.6-1703 (observed for the first time simultaneously in the energy range $0.3-100 \mathrm{keV}$ during a bright flare).
\end{abstract}

The Extreme sky: Sampling the Universe above $10 \mathrm{keV}$

October 13-17 2009

Otranto (Lecce) Italy

* Speaker. 


\section{Supergiant Fast X-ray Transients}

Supergiant Fast X-ray Transients (SFXTs) are transient X-ray sources in binary systems composed of a compact object and a blue supergiant companion. Although some of them were discovered before the INTEGRAL satellite launch in 2002, this new class of High Mass X-ray Binaries (HMXBs) was recognized only after several new peculiar X-ray transients had been found because of the Galactic plane survey performed by INTEGRAL ([18], [10]). The members of this new class of sources display apparently short outbursts (as observed with INTEGRAL), characterized by a few hour duration flares and by a high dynamic range $(1,000-100,000)$ between the quiescence $\left(10^{32} \mathrm{erg} \mathrm{s}^{-1}\right)$ and the flare peak $\left(10^{36}-10^{37} \mathrm{erg} \mathrm{s}^{-1}\right)$.

The spectra are very similar to those of accreting pulsars, displaying a flat power law spectrum below $10 \mathrm{keV}$, and a high energy cutoff in the range 10-30 keV (e.g. [26, 20]). This spectral similarity suggests that all SFXTs host a neutron star as compact object, although only in four sources pulsations have been discovered: AX J1841.0-0536 ( $P_{\text {spin }} \sim 4.7 \mathrm{~s}$, [1]), IGR J11215$5952\left(P_{\text {spin }} \sim 187 \mathrm{~s}\right.$, [27]), IGR J16465-4507 ( $P_{\text {spin }} \sim 228 \mathrm{~s}$, [9]) and IGR J18483-0311 ( $P_{\text {spin }} \sim$ $21 \mathrm{~s}$, [19]). The distribution of their orbital periods (measured in 5 SFXTs) ranges from 3.3 days (IGR J16479-4514, [7]) to 165 days (in IGR J11215-5952, [20, 21, 13, 15]). Periodically recurrent outbursts have been observed in two sources, IGR J11215-5952 [20] and IGR J18483-0311 ([8, 19]), indicative of outbursts triggered near the periastron passage in a highly eccentric orbit.

The physical mechanism responsible for their peculiar transient X-ray emission is still an open issue (see [23] for a review of the different possibilities). The main proposed mechanisms are related to the structure of the supergiant wind, accreting onto the compact object ([32, 31, 21, $11,4]$ ) and/or to the neutron star magnetic field (suggested to be magnetar-like) and its (long) spin period $([6,2])$. These different scenarii need a comparison with the SFXTs observative properties, which were, on the other hand, largely unknown before our monitoring campaign with Swift/XRT, which started in October 2007. The goal of this campaign was to address several crucial issues, in particular the source status outside the bright flaring activity: are SFXTs in quiescence (no accretion) when they are not in bright flaring activity? or are they still in accretion, but at a much lower level, too low to be detected by INTEGRAL? which is the duty cycle of their transient X-ray emission?

The rapid flaring variability together with the association with a massive companion is indicative of the fact that SFXTs are wind accretors, similar to the classical persistent HMXBs with supergiant companions (like Vela $\mathrm{X}-1$ ) of the same spectral type. One of the main open issues is the link between these two subclasses of massive binaries. Both kind of binaries are composed by a compact object (likely a neutron star) and by an OB supergiant companion with similar spectral type. For this reason, it was proposed that different orbital parameters ([11]) could probably separate the two subclasses: circular and narrow orbits in persistent HMXBs, while wide and eccentric orbits in SFXTs. On the other hand, the recent discovery of the orbital period of 3.3 days in the SFXT IGRJ16479-4514 [7], is puzzling, because it implies a narrower orbit than that shown by several persistent HMXBs. This seems to suggest that at least in a few members of the class, the orbit is not the main parameter which separates the transient from the persistent behaviour.

Before our monitoring, the broad band spectra $(0.3-100 \mathrm{keV})$ were obtained only from not simultaneous observations along the whole energy range, thus the suggested spectral similarity 
between SFXTs and persistently accreting X-ray pulsars needed to be tested by simultaneous wide band observations. The duration of the outburst phase was unknown, except in the case of the periodic SFXT IGR J11215-5952 [13], where our monitoring with Swift demonstrated that the outburst lasts a few days, instead of only a few hours (as previously observed by INTEGRAL during the brightest flares). Other crucial properties, like the distribution of the neutron star magnetic fields, the spin and the orbital periods, were completely unknow, as well.

\section{Swift contribution}

We performed a long-term monitoring campaign with Swift/XRT of a sample of four SFXTs, XTE J1739-302, IGR J17544-2619 (the two prototypes of the class), IGR J16479-4514 and the X-ray pulsar AX J1841.0-0536, in order to try to address all these important and open issues. The campaign strategy consists of 2 or 3 XRT pointings per source per week (about $\sim 1 \mathrm{ks}$ each) to frequently monitor the source status. Given the structure of the observing plan, this monitoring can be considered as a casual sampling of the source light curves at a resolution of about $\sim 4$ days. We were mainly interested in the monitoring of the long-term properties, to get a census of all the outbursts (even the fainter ones, not triggering the Swift/BAT), to monitor the onset of each new outburst and to follow the whole outburst duration with more frequent subsequent observations (in this respect the Swift flexibility is a crucial property), and to get truly simultaneous wide band spectra during bright flaring activity. Our monitoring campaign (which is still on-going) has completely changed the previous view of this transient sources $([22,14,24,25,16])$.

Swift observations have demonstrated that SFXTs spend most of their life still accreting matter even outside bright flaring activity, with an intermediate level of X-ray emission at $10^{33}$ $10^{34} \mathrm{erg} \mathrm{s}^{-1}$, large flux variability (at least one order of magnitude) and an absorbed power law spectrum below $10 \mathrm{keV}$ (photon index of 1-2, or hot black body temperatures of 1-2 keV). Besides the bright outbursts (detected also with BAT) and the intermediate level of X-ray emission, several $3 \sigma$ upper limits were also measured, either because the source was faint or due to a very short exposure time because of the interruption by a gamma-ray burst. In order to get an as uniform as possible subsample for the "non-detections" state, we excluded all observations with a net exposure below $900 \mathrm{~s}$. An exposure of $900 \mathrm{~s}$ corresponds to $2-10 \mathrm{keV}$ flux limits of $\sim 1-3 \times 10^{-12} \mathrm{erg}$ $\mathrm{cm}^{-2} \mathrm{~s}^{-1}(3 \sigma)$, depending on the source (assuming the best fit absorbed power law model for the intermediate state of each source). Then, a duty cycle of inactivity (IDC) was defined as the time fraction each source spends undetected down to a flux limit of $1-3 \times 10^{-12} \mathrm{erg} \mathrm{cm}^{-2} \mathrm{~s}^{-1}$ (which means an upper limit to the time spent in quiescence). The IDCs we obtained during the campaign with Swift/XRT are the following: $17 \%$ (IGR J16479-4514), $28 \%$ (AX J1841.0-0536), $39 \%$ (XTE J1739-302) and $55 \%$ (IGR J17544-2619). In the eclipsing SFXT IGR J16479-4514 a main contribution to the IDC comes from the X-ray eclipses, hence the above $17 \%$ is in fact an upper limit to the true quiescent time. To summarize, the quiescence in these transients is a rarer state [16] than what previously thought based only on INTEGRAL observations. The lowest luminosity level we could observe with Swift/XRT, obtained accumulating all data for which no detections were obtained as single exposures [16], is reached in XTE J1739-302 $\left(6 \times 10^{32} \mathrm{erg} \mathrm{s}^{-1}\right.$, $2-10 \mathrm{keV})$ and in IGR J17544-2619 $\left(3 \times 10^{32} \mathrm{erg} \mathrm{s}^{-1}\right)$. 
We observed the broad band simultaneous spectra (XRT together with BAT) during 8 bright flares observed from 3 of the 4 monitored sources. The best fits could be obtained with Comptonized models (COMPTT or BMC in XSPEC) or with an absorbed flat power law model with high energy cutoff around 10-30 keV (see [14, 24, 25]). This spectral shape, and in particular the spectral cutoff, is compatible with a neutron star magnetic field of $\sim 10^{12} \mathrm{G}$ [3], although no cyclotron lines have been detected yet in these four sources. We found evidence for variable absorbing column densities, both in the same source (XTE J1739-302) and different outbursts and within the same outburst, indicative of dense clouds of matter composing the supergiant wind passing towards the line of sight.

Another crucial finding of our monitoring campaign is that the SFXTs bright and short flares (a few hour long) are part of a longer outburst phase lasting days [24], as already found in the periodic SFXT IGR J11215-5952 [13].

The first optical/UV observations performed with UVOT simultaneously to our Swift/XRT monitoring of the SFXTs revealed a possible hint of an UV flaring activity simultaneously to the $\mathrm{X}$-ray bright flares in XTE J1739-302. However this findings needs confirmation because it is a less than $3 \sigma$ result [16].

\section{SAX J1818.6-1703}

SAX J1818.6-1703 is a Supergiant Fast X-ray Transient associated with a B0.5Iab type companion located at $2.1 \mathrm{kpc}$ ([30]). It triggered the Swift/BAT on 2009 May 5 at 14:03:27 UT, and after the BAT trigger, the whole outburst evolution and the decline phase could be monitored with XRT. The time resolved spectroscopy with XRT did not result in variability of the spectral parameters in the $1-10 \mathrm{keV}$ range, within the uncertainties. No variability in the absorbing column density could be detected along the outburst, as well.

Simultaneous BAT and XRT spectra, between 138 and 937 s since the BAT trigger, allowed us to obtain the first broad band X-ray spectrum of this source during an outburst, with a joint fit in the $1-10 \mathrm{keV}$ and $14-150 \mathrm{keV}$ energy bands for XRT and BAT, respectively. This wide band X-ray spectrum, highly absorbed $\left(N_{\mathrm{H}} \sim 5-7 \times 10^{22} \mathrm{~cm}^{-2}\right)$, is well deconvolved with models like power laws with high energy cutoffs (CUTOFFPL in XSPEC), or Comptonization models [COMPTT [29] or the BMC model [28] in XSPEC]. Adopting the COMPTT model, the properties of the Comptonizing corona could be constrained well with an electron temperature $k T_{e} \sim 5-7 \mathrm{keV}$ and an optical depth $\tau \sim 10$ (in a spherical geometry). These properties are reminiscent of the $\mathrm{X}$-ray spectral shape of the prototype of the SFXT class, XTE J1739-302.

\section{IGR J18483-0311}

The SFXT IGR J18483-0311 is an X-ray pulsar ( 21 s, [19]) and is the second member of the class where periodically recurring outbursts have been discovered with a period of $18.55 \pm$ 0.03 days $([8,19])$, which is very likely the orbital period of the system. It is associated with a blue supergiant (B0.5Ia) at a distance of 3-4 kpc [12].

We monitored with Swift an entire orbital phase (28 days for a total on-source exposure of $\sim 44 \mathrm{ks}$ ), starting on 2009 June 11 with 2 ks per day ([17]). The XRT observations shows a highly 
modulated light curve with two maxima, separated by a time interval consistent with the orbital period of $\sim 18.5$ days. A lower limit of 1200 to the dynamical range can be obtained from the observed light curve. The different duration of the two outburst peaks monitored with Swift is likely resulting from both a different sampling and a high intrinsic X-ray variability. The second peak has a duration of several days, as previously observed by INTEGRAL [19].

We interpret the modulation of the light curve with the orbital phase as wind accretion along a highly eccentric orbit. These observations allow to constrain the different mechanisms proposed to explain the nature of the new class of SFXTs. Applying the new clumpy wind model for blue supergiants developed by [4] to the evolution of the observed X-ray light curve, we found that, assuming an eccentricity of $e=0.4$, the $\mathrm{X}$-ray emission from this source can be explained in terms of the accretion from a spherically simmetric clumpy wind, composed by clumps with masses ranging from $10^{18} \mathrm{~g}$ to $5 \times 10^{21} \mathrm{~g}$ (see Ducci et al., these proceedings for details of the model).

\section{References}

[1] Bamba, A., Yokogawa, J., Ueno, M., et al., 2001, PASJ, 53, 1179

[2] Bozzo, E., Falanga, M., Stella, L., 2008, ApJ, 683, 1031

[3] Coburn, W., Heindl, W. A., Rothschild, R. E. et al., 2002, ApJ, 580, 394-412

[4] Ducci, L., Sidoli, L., Mereghetti, S. et al., 2009, MNRAS, 398, 2152-2165

[5] Ducci, L., Sidoli, L., Mereghetti, S., et al., 2009, these proceedings

[6] Grebenev, S.A., and Sunyaev, R. A., Astronomy Letters, 33, 149-158

[7] Jain, C., Paul, B., and Dutta, A., 2009, MNRAS, 397, L11

[8] Levine, A. M., Corbet, R., The Astronomer's Telegram 940

[9] Lutovinov, A., Revnivtsev, M., Gilfanov, M., et al. 2005, A\&AS, 444, 821-829

[10] Negueruela, I., Smith, D.M., Reig, P., et al. 2006a, in ESA Special Publication, ed. A.Wilson, Vol. 604, 165-170

[11] Negueruela, I., Torrejon, J.M., Reig, P., et al., 2008, AIPC, 1010, 252

[12] Rahoui, F., Chaty, S., 2008, $A \& A, 492,163$

[13] Romano, P., Sidoli, L., Mangano, V. et al., 2007a, $A \& A, 469$, L5

[14] Romano, P., Sidoli, L., Mangano, V., et al., 2008, ApJL, 680, L137-L140

[15] Romano, P., Sidoli, L., Cusumano, G., et al., 2008, ApJ, 696, 2068-2074

[16] Romano, P., Sidoli, L., Cusumano, G., et al., 2009, MNRAS, 399, 2021-2032

[17] Romano, P., Sidoli, L., Ducci, L., et al., 2009, MNRAS in press (arXiv:0909.5109)

[18] Sguera, V., Barlow, E.J., Bird, A.J., et al. 2005, $A \& A, 4$ 444, 221

[19] Sguera, V., Hill, A. B., Bird, A. J., et al., 2007, $A \& A, 4$ 467, 249

[20] Sidoli, L., Paizis, A., \& Mereghetti, S., 2006, A\&A, 450, L9

[21] Sidoli, L., Romano, P., Mereghetti, S., et al., 2007, A\&A, 476, 1307 
[22] Sidoli, L., Romano, P., Mangano, V., 2008, ApJ, 687, 1230

[23] Sidoli, L., 2008, Proc. of the COSPAR Assembly 2008, 37, 2892

[24] Romano, P., Sidoli, L., Mangano, V., et al., 2008, ApJ, 690, 120-127

[25] Sidoli, L., Romano, P., Esposito, P., et al., 2009, MNRAS, 400, 258-262

[26] Smith, D.M., Main, D., Marshall, F., et al., 1998, ApJL, 501, L181

[27] Swank, J.H., Smith, D.M., Markwardt, C.B., 2007, The Astronomer's Telegram, 999

[28] Titarchuk, L., Mastichiadis, A., Kylafis, N. D., 1996, A\&AS, 120, 171

[29] Titarchuk, L., 1994, ApJ 434, 570-586

[30] Torrejon, J.M., Negueruela, I., Smith, D. et al. 2009, $A \& A$ in press (arXiv0910.5603)

[31] Walter, R., \& Zurita Heras, J., 2007, A\&A, 476, 335

[32] in't Zand, J.J.M., 2005, A\&A, 441, L1 